\title{
Pre-Operative Interictal Discharge Patterns and Magnetic Resonance Imaging Findings Affect Prognosis of Temporal Lobe Epilepsy Surgery
}

\author{
Weining Ma ${ }^{a} \quad$ Chun Lib Liping Liua Shaoyi Lia Yunhui Liua \\ a Department of Neurosurgery, Shengjing Hospital Affiliated to China Medical University, Shenyang, China; \\ ${ }^{b}$ Department of Pediatric Neurology, Shengjing Hospital Affiliated to China Medical University, Shenyang, China
}

\section{Keywords}

Temporal lobe epilepsy · Interictal discharge $\cdot$ Magnetic resonance imaging

\begin{abstract}
Objective: This study investigated whether pre-operative interictal discharge patterns detected by electroencephalogram (EEG) and magnetic resonance imaging (MRI) findings affect the surgical prognosis in temporal lobe epilepsy (TLE) patients. Methods: A retrospective analysis of 115 cases of patients with refractory TLE was carried out from October 2010 to 2014 based on the classification of pre-operative interictal discharge patterns in EEG and MRI findings. The patients were followed up for 4 years after surgery. The ILAE method was used to assess differences in seizure-free rate among different types of interictal discharge pattern as well as in MRI findings. Results: A total of 115 cases were classified according to interictal discharge patterns in EEG, including normal cases, unilateral anterior discharge, unilateral multi-region discharge, and bilateral discharge. MRI findings were classified into negative results and positive results. Unilateral anterior and bilateral discharges showed statistically significant differences in post-operative seizure-free rates $(p<0.001)$. MRI-positive cases showed good overall post-
\end{abstract}

operative outcome, irrespective of interictal discharge pattern in the EEG, whereas MRI-negative cases showed good overall prognosis if the interictal discharge pattern in EEG occurred in the unilateral anterior region. Conclusion: If the pre-operative interictal discharge pattern in EEG is confined to the unilateral anterior region, prognosis is good. If there are abnormalities in MRI findings, post-operative prognosis is good, regardless of pre-operative interictal discharge patterns in EEG. Surgical intervention is highly recommended for TLE patients with normal MRI findings and interictal discharge confined to the unilateral anterior region.

(c) 2019 S. Karger AG, Basel

\section{Introduction}

Epilepsy is a common neurological disorder, affecting approximately 70 million patients worldwide; $90 \%$ of cases are reported in the under-developed areas [1]. The global incidence of epilepsy ranges from 6.38 to $7.60 \%$ [2], and the number of new cases reported annually is $67.77 / 100,000$ people. The incidence in China is

W.M. and C.L. contributed equally to this work.

\section{KARGER}

(c) 2019 S. Karger AG, Basel

E-Mail karger@karger.com

www.karger.com/ene
Yunhui Liu, MD, $\mathrm{PhD}$

Department of Neurosurgery

Shengiing Hospital Affiliated to China Medical University

36 Sanhao Street, Shenyang 110004 (China)

E-Mail yunhui_cmu@163.com 
$1.19-6.70 \%$ [3]. In some epilepsy patients, pharmacological intervention is ineffective; these cases are defined as drug-resistant epilepsy [4], with temporal lobe epilepsy (TLE) accounting for about 70\% [5]. Although surgery is effective for treating TLE, it does not control seizure onset in all patients [6-8]; this may depend on the type of TLE [9], disease duration, and electroencephalogram (EEG) and magnetic resonance imaging (MRI) results [10]. Pre-operative bilateral discharge in EEG has been linked to poor prognosis following surgery [11]. The presence of lesions in MRI scans predicted seizures in $62-80 \%$ of cases; when the MRI results did not show a clear abnormality, the rate of seizures was $36 \%$ [12]. It is therefore important to establish a method for predicting the prognosis of TLE to improve patient outcome.

The present study retrospectively analyzed pre-operative EEG and MRI data of 115 cases of TLE in the 1- to 4 -year follow-up periods. We examined the type of preoperative interictal discharge pattern in EEG and its relationship with MRI findings. We also compared changes in EEG before and after surgery, and investigated the relationship between pre-operative interictal discharge patterns and MRI findings and post-surgery prognosis.

\section{Materials and Methods}

\section{Clinical Case Collection}

A total of 115 cases of refractory TLE were treated at the Department of Functional Neurosurgery at China Medical University affiliated with Shengjing Hospital from October 2010 to 2014. The pre-operative EEG, MRI examination results, and EEG records and seizure control conditions of patients from post-operative year 1-4 follow-up periods were collected. The study was approved by the Ethics Committee of China Medical University affiliated with Shengjing Hospital and patients provided written informed consent for the use of their data.

\section{Pre-Operative Assessment}

Each patient underwent a comprehensive pre-operative assessment that included scalp video EEG monitoring (Fig. 1a-c), intracranial EEG in cases where the scalp EEG was unclear (Fig. 1d); head and hippocampus MRI (Fig. 3a-c, e-h), in which suspected hippocampal sclerosis patients underwent magnetic resonance spectroscopy examination (Fig. 3d); head positron emission tomography-computed tomography/MRI (Fig. 3i, j); and neuropsychological evaluation. The interictal discharge pattern EEG was classified into the following 4 categories according to the region of discharge: (1) normal; (2) unilateral discharge in the anterior region (temporal and frontal regions; Fig. 1b); (3) unilateral discharge in multiple regions (cumulative region $\geq 3$ regions; Fig. 1a); and (4) bilateral discharge (including bilateral temporal regions; Fig. 1c). At least $24 \mathrm{~h}$ of interictal EEG should be recorded, which must include both waking and sleeping periods.
MRI findings were classified as follows: (1) normal (negative; Fig. 3a); (2) hippocampal sclerosis (Fig. 3c, d); (3) unilateral hippocampal swelling (Fig. 3b); (4) tumors (Fig. 3e); (5) vascular diseases, including vascular malformation and cavernous hemangioma (Fig. 3h); (6) encephalomalacia (Fig. 3f), including those induced by trauma and cerebrovascular disease; and (7) focal cortex dysplasia (Fig. 3g).

\section{Case Selection Criteria}

A diagnosis of TLE was made based on EEG results and symptomology during the epileptic episode. The case selection criteria were as follows: (1) scalp EEG or intracranial electrode EEG originating from the temporal lobe; (2) positive MRI, with lesions located in the temporal lobe; (3) lesions in the encephalomalacia associated with the temporal lobe; and (4) cases meeting the diagnostic criteria for drug-refractory epilepsy.

\section{Surgery Methods}

The following 3 types of surgery were performed: (1) anterior temporal lobe and hippocampal amygdala resection; (2) temporal lobe resection (hippocampal retention); and (3) resection of lesion (e.g., tumor, vascular malformation, and encephalomalacia). All intracranial EEG monitoring was performed intraoperatively except when there were pre-existing intracranial electrodes. The purpose of EEG monitoring was to assess the condition of the hippocampus (Fig. 2b) and the involvement of the temporal cortex (Fig. 2c) and ipsilateral frontal lobe (Fig. 2d). EEG monitoring was performed using a 32-lead-shaped subdural electrode that was placed on the ipsilateral frontal and temporal lobes following midline incision; it required that the tip of the electrode be rolled up into the skull base and medial temporal lobe structures (Fig. 2a). For cases with no definite lesions in the temporal cortex, the resection area usually did not exceed Labbe's vein.

\section{Post-Operative Treatment and Follow-Up}

Post-operative antiepileptic drugs were administered for continuous treatment. In general, the pre-operative drug was maintained and serum drug concentrations were regularly monitored. Follow-up evaluations at 3 and 6 months and 1, 2, 3, and 4 years were conducted by telephone or by EEG monitoring at the hospital. Post-operative evaluation of seizures was based on the International League Against Epilepsy method [13]. Seizures were defined as being completely controlled in cases meeting the Class 1 requirement.

\section{Statistical Analysis}

Trends in pre- and post-operative interictal discharge patterns at different follow-up time points were analyzed. Post-operative seizure-free rates were calculated based on interictal discharge patterns. The relationship between MRI findings and interictal discharge patterns was evaluated statistically. Changes in interictal discharge patterns in EEG before and after surgery were analyzed, and post-operative seizure-free rates were calculated based on the MRI findings. The Chi-square test for independence was used to compare differences in post-operative seizure-free rates between cases of unilateral anterior and bilateral discharge, as well as differences in seizure-free rates between MRI-negative and -positive cases. $p<0.05$ was considered statistically significant. 

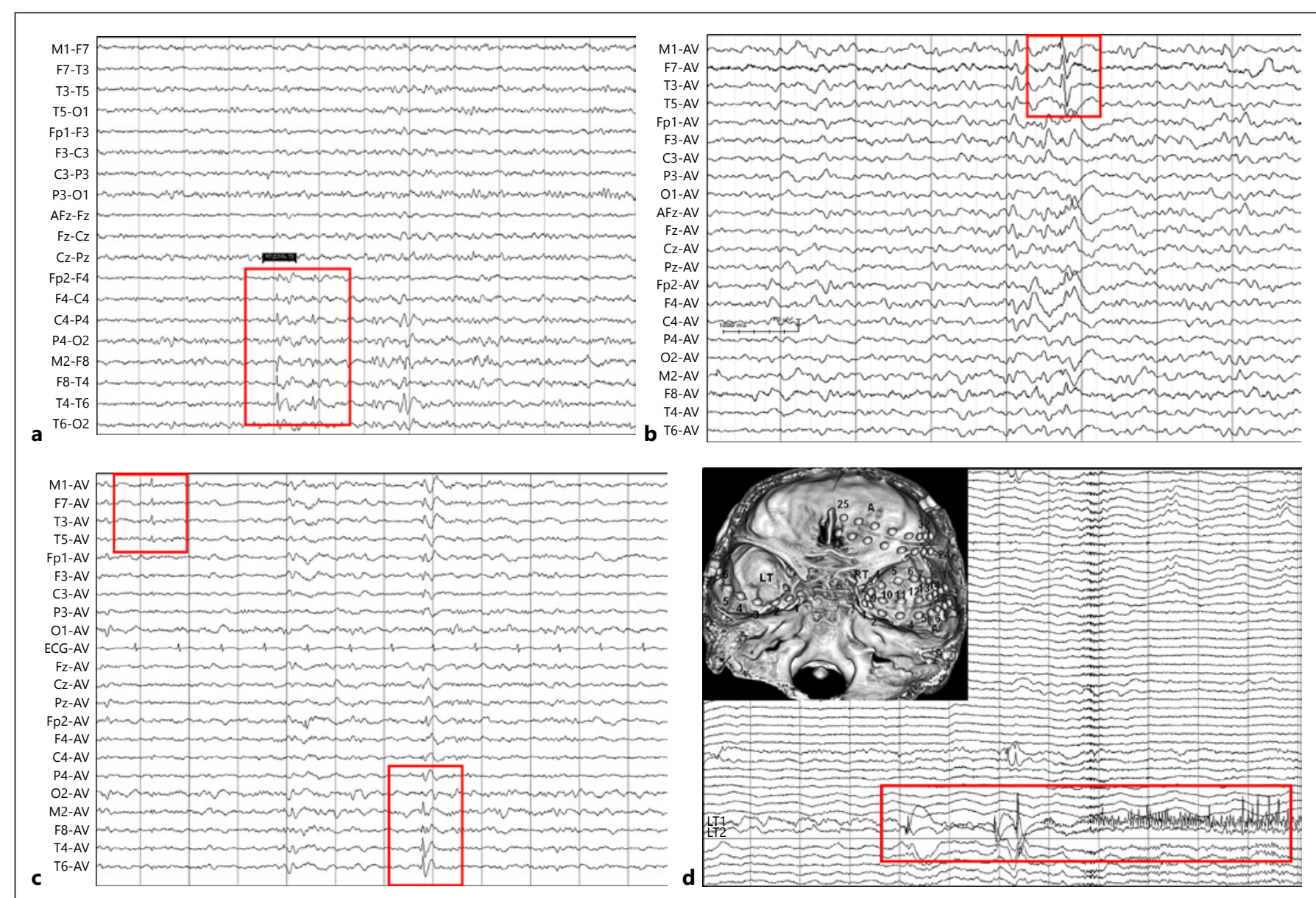

Fig. 1. Pre-operative discharge patterns in EEG. a Observable interictal multi-region discharges on the right. b Observable interictal anterior discharges on the left (left temporal region). c Noticeable interictal bilateral non-synchronous discharges (involving bilateral temporal regions). d Electrode burial at bilateral temporal

\section{Results}

\section{Characteristics of the Study Population}

The study population consisted of 115 patients, including 59 males (51.3\%) and 56 females (48.7\%), ranging in age from 2 to 62 years with a mean age of $30.8 \pm 12.6$ years. A total of 51 patients (44.3\%) had epilepsy in the left temporal lobe, and $64(55.7 \%)$ in the right temporal lobe. There were 35 cases (31.2\%) with normal MRI results and $80(68.8 \%)$ with clear abnormalities in the MRI scan, including hippocampal sclerosis $(n=29,26.6 \%)$, unilateral hippocampal swelling $(n=8,6.4 \%)$, tumor $(n=18,14.7 \%)$, vascular disease $(n=7,6.4 \%)$, encephalomalacia $(n=13$, $11.0 \%)$, and focal cortical dysplasia $(n=5,3.7 \%)$. There were 5 cases with normal EEG (4.3\%); EEG abnormalities and left frontal subdural regions. Three-dimensional computed tomography synthetic chart (a) of the electrode. The intracranial electrode in the EEG showed that the seizure originated from the left medial LT1 and LT2 electrode contacts.

included unilateral anterior discharges $(n=52,45.2 \%)$, unilateral multi-region discharges $(n=7,6.7 \%)$, and bilateral discharges $(n=51,44.3 \%)$. Surgical interventions included anterior temporal lobe and hippocampal amygdala resection $(n=101 ; 87.8 \%)$, temporal lobe resection $(n=8,7.0 \%)$, and lesion resection $(n=6,5.3 \%) ; 6$ of the 115 cases were lost to follow-up (Table 1).

\section{EEG Interictal Discharge Patterns before and after Surgery}

The proportions of pre-operative normal EEG among post-operative EEG at 3 and 6 months and 1,2, 3, and 4 years were $4.3,42.3,56.3,57.9,69.2,60.0$, and $63.6 \%$, respectively. For unilateral anterior discharge, the proportions were $45.2,44.2,31.3,21.1,17.9,12.0$, and $27.3 \%$, re- 


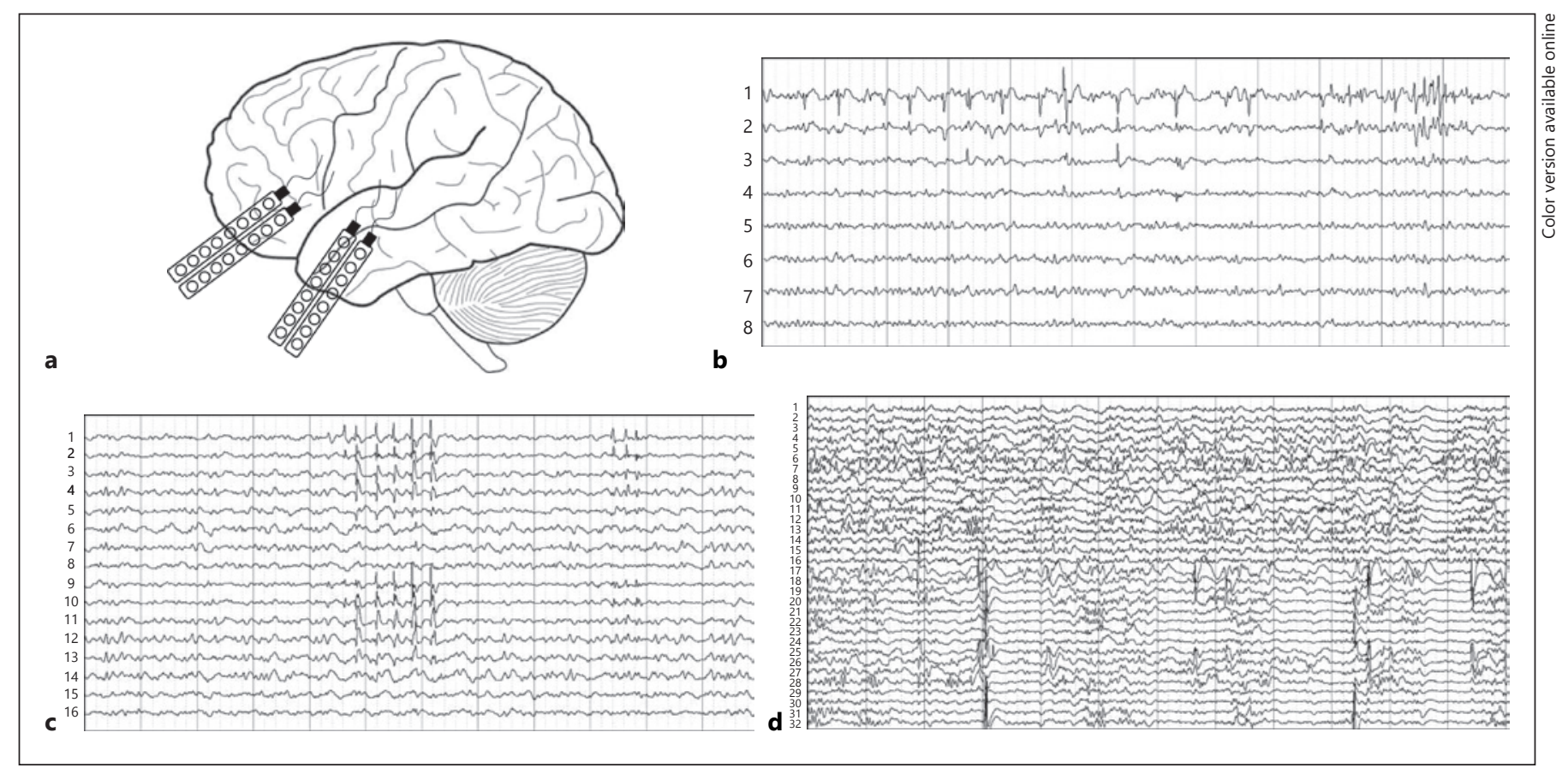

Fig. 2. Intraoperative intracranial electrode monitoring. a Scheme of intracranial electrode monitoring mode. b Hippocampal discharge mainly from the no. 1 contact spot. c Discharge from the

spectively. For unilateral multi-region discharge, the proportions were $6.2,0,6.2,5.2,7.7,4.0$, and $0 \%$, respectively. For bilateral discharge, the proportions were 44.3, 13.5, 6.2, 15.8, 5.2, 8.0, and 9.1\%, respectively (Fig. $4 \mathrm{a}$ and online suppl. Table 1; for all online suppl. material, see www.karger.com/doi/10.1159/000501002). These results indicate that post-operative EEG patterns progressed to normal and unilateral anterior confined discharge patterns, suggesting that they are associated with post-operative control of epilepsy.

\section{Relationship between MRI and Interictal EEG}

For patients with normal MRI results, the pre-operative interictal EEG showed unilateral anterior discharge in $48.6 \%$ of cases and bilateral discharge in $51.4 \%$ of cases. For hippocampal sclerosis, $58.6 \%$ showed unilateral anterior discharge, $6.9 \%$ unilateral multi-region discharge, and $34.5 \%$ bilateral discharge. Unilateral hippocampal swelling was associated with unilateral anterior discharge (50.0\%) and bilateral discharge (50\%). Among patients with tumors, $11.1 \%$ were normal, $38.9 \%$ had unilateral anterior discharge, $16.7 \%$ unilateral multi-region discharge, and $33.3 \%$ bilateral discharge. For patients with vascular diseases, $14.2 \%$ were normal, $42.9 \%$ showed unilateral anterior discharge, and $42.9 \%$ bilat- new cortex under the temporal lobe. $\mathbf{d}$ The temporal lobe had frequent discharges (17-21 contact points), but no abnormal discharges (1-16 contact points) were observed in the frontal lobe.

eral discharge. Among normal cases of encephalomalacia, $15.4 \%$ were normal, $15.4 \%$ had unilateral anterior discharge, $15.4 \%$ unilateral multi-region discharge, and $53.8 \%$ bilateral discharge. For patients with focal cortical dysplasia, $40.0 \%$ had unilateral anterior discharge and $60.0 \%$ had bilateral discharge (Fig. $4 \mathrm{~b}$ and online suppl. Table 2). For post-operative follow-up time points, the overall EEG changes in MRI cases were evaluated. The overall trend was a progression to normal and confined unilateral anterior discharge. However, there was a high proportion of bilateral discharge among MRI-negative cases (Fig. 4c and online suppl. Table 3). In MRI-positive cases, the post-operative EEG was likely to be normal or show confined unilateral anterior discharge (Fig. 4c-i and online suppl. Table 3). This suggests that the interictal discharge pattern of the bilateral discharge is associated with poor prognosis in epilepsy.

\section{Status of Post-Operative Seizure Control}

The overall post-operative seizure-free rates at 1, 2, 3, and 4 years were $90.2,79.8,75.2$, and $72.5 \%$, respectively (online suppl. Table 4). Based on pre-operative interictal EEG patterns, seizure-free rates at the 4-years follow-up periods were as follows: normal pattern, $100 \%$; unilateral anterior discharge pattern, 96.1, 92.2, 88.2, and 86.3\%; 

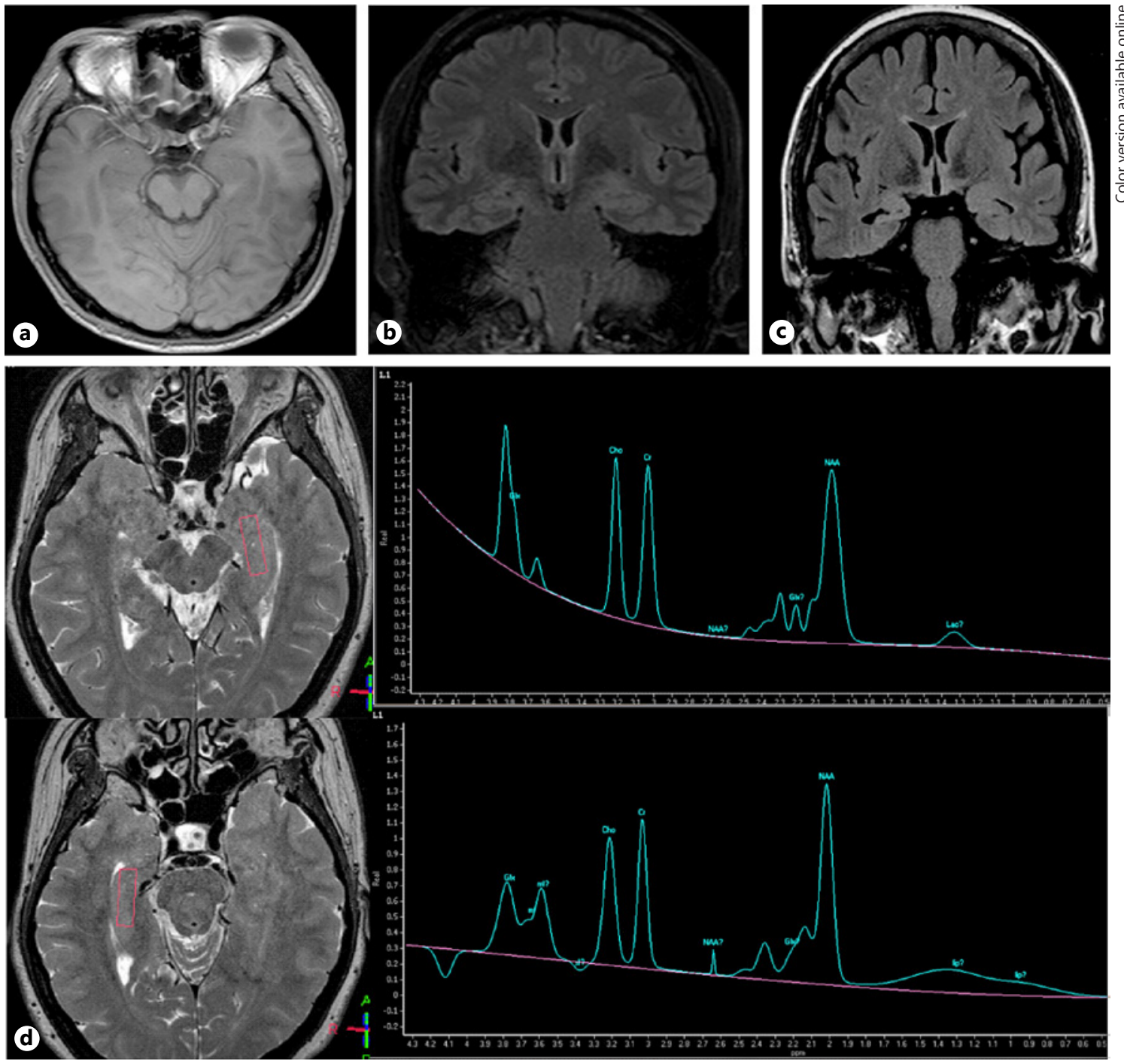

Fig. 3. Pre-operative imaging examination findings in EEG. a Normal in MRI (T1-sequence). b Left hippocampal swelling (T1-sequence). c Right hippocampal sclerosis (T2-flair-sequence). d Left hippocampal sclerosis (MRS: Left hippocampal NAA/[Cho + Cr] ratio was lower than the right one). e Low-grade gliomas in right medial temporal lobe (T2-sequence). f Right temporal lobe traumatic encephalomalacia (T2-sequence). g Left temporal lobe FCD (T2-flair-sequence). $\mathbf{h}$ Left temporal lobe cavernous hemangioma (T2-flair-sequence). i Low metabolism in left temporal lobe (PETCT scan). $\mathbf{j}$ Low metabolism in left temporal lobe (PET-MRI scan).

(Figure continued on next page.)

unilateral multi-region discharge pattern, 71.4, 57.1, 57.1, and 57.1\%; bilateral discharge pattern, 69.6, 67.4, 60.9, and $56.5 \%$ (Fig. 5a and online suppl. Table 5). There was a significant difference in post-operative seizure-free rates between bilateral and unilateral anterior discharge groups ( $p<0.001$; Fig. $5 d$ ). Seizure-free rates were 58.8, $50.0,44.1$, and $38.2 \%$ among MRI-negative cases, respectively and 94.7, 93.3, 89.3, and 88.0\% among MRI-positive cases, respectively at the 4 -years follow-up periods (Fig. 5b and online suppl. Table 4). There was a significant difference in post-operative seizure-free rates between
MRI-positive and MRI-negative cases ( $p<0.001$; Fig. 5e). In MRI-positive cases, the post-operative seizure-free rates were also evaluated based on the results of MRI classification (Fig. 5c and online suppl. Table 6).

\section{Discussion}

At the 4-year follow-up of 109 cases of TLE, the postoperative first year overall effect was highest at $90.2 \%$. The seizure-free rate was more than $80 \%$ in Deleo et al. 

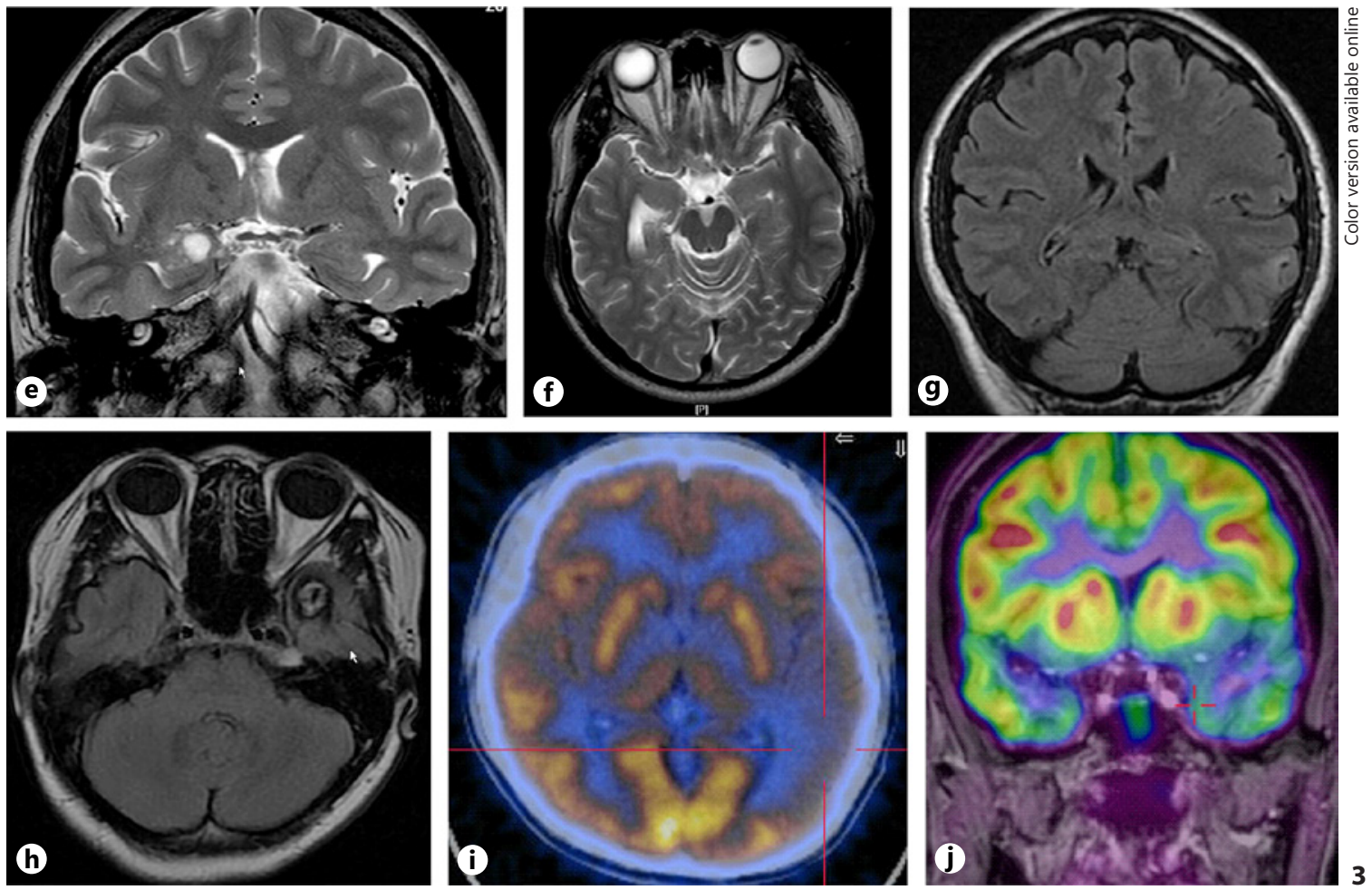

Table 1. The demographic of the cases

\begin{tabular}{|c|c|}
\hline The total number of cases & 115 \\
\hline The number of lost to follow-up, years & 6 \\
\hline Age, years & 2-62 (AVE $30.8 \pm 12.6)$ \\
\hline \multicolumn{2}{|l|}{ Gender } \\
\hline Male & 59 \\
\hline Female & 56 \\
\hline \multicolumn{2}{|l|}{ Origin of temporal lobe epilepsy } \\
\hline Left & 51 \\
\hline Right & 64 \\
\hline \multicolumn{2}{|l|}{ MRI } \\
\hline Normal & 35 \\
\hline Hippocampal sclerosis & 29 \\
\hline Unilateral hippocampal swelling & 8 \\
\hline Tumors & 18 \\
\hline Vascular diseases & 7 \\
\hline Encephalomalacia & 13 \\
\hline FCD & 5 \\
\hline \multicolumn{2}{|l|}{ EEG } \\
\hline Normal & 5 \\
\hline Unilateral anterior discharge & 52 \\
\hline Unilateral multi-region discharge & 7 \\
\hline Bilateral discharge & 51 \\
\hline \multicolumn{2}{|l|}{ Operation types } \\
\hline $\begin{array}{l}\text { Anterior temporal lobe and } \\
\text { hippocampus-amygdala resection }\end{array}$ & 101 \\
\hline Temporal lobe resection & 8 \\
\hline Lesionectomy & 6 \\
\hline
\end{tabular}

FCD, focal cortical dysplasis; MRI, magnetic resonance imaging; EEG, electroencephalogram.
[14] research including 213 TLE patients with at least 2 years follow-up. The seizure-free rate 4 years after surgery was $72.5 \%$ according to our results, indicating that surgery is an effective treatment for TLE. As the followup time increased, the seizure-free rate of seizures after TLE decreased, which is consistent with the results of other clinical studies [7]. Although the reason for this trend is unclear, it may be attributed to stimulation during surgery, temporal plus epilepsy, other potentially epileptogenic foci, past history of status epilepticus, and preoperative intracranial electroencephalographic recording [15]. For TLE surgery, most cases could be localized by scalp interictal discharge and MRI manifestations, and the consistency between interictal discharge and MRI was usually very high [16]. In cases where the scalp EEG is unable to identify the origin of the epilepsy, the intracranial electrode should be used to clarify the position and site of resection. In 16 of the 115 cases, an operation to bury the subdural grid electrode was performed. It is now also possible to bury stereotactic intracranial electrodes to analyze the origin of temporal lobe seizures [17]. When there was a clear abnormality in the MRI results that was highly correlated with the scalp EEG readout, surgical intervention should be considered. When the MRI scan showed a normal condition but the EEG and ictal symptoms suggested a case of mesial TLE, 


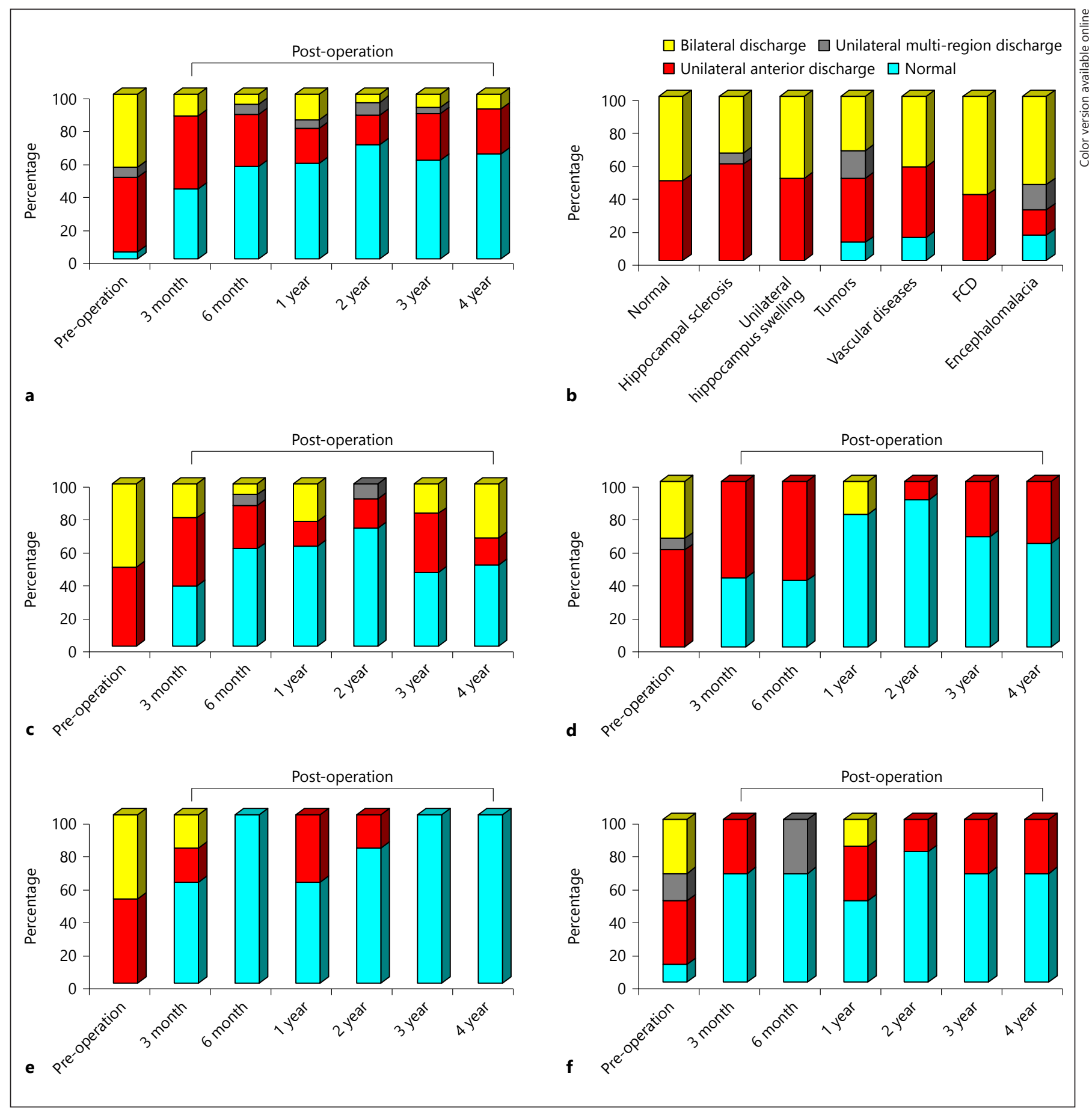

Fig. 4. Trend of changes in the proportion of interictal discharge patterns in the EEG before and after surgery. a Statistical analysis based on interictal discharges in the EEG. b Proportion of interictal discharge patterns in the EEG for different pre-operative MRI

results. c Cases with normal MRI. d Cases of hippocampal cirrhosis. e Cases with unilateral hippocampal swelling. $\mathbf{f}$ Cases with tumors. $\mathbf{g}$ Cases showing intracranial vascular diseases. $\mathbf{h}$ Cases of encephalomalacia. i Cases with FCD.

(Figure continued on next page.) 


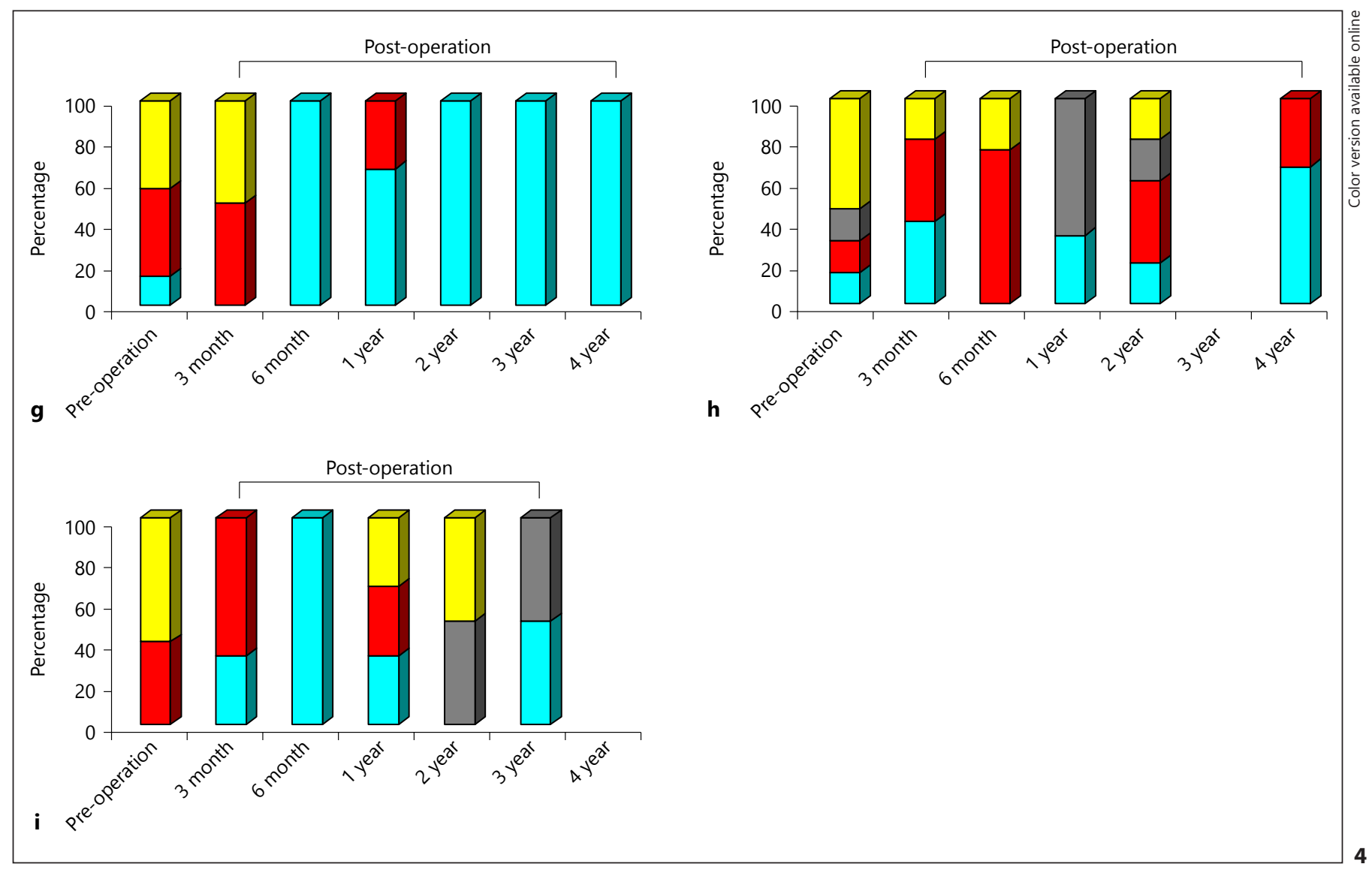

we used direct surgical resection. The resection of the hippocampus largely depended on the characteristics of mesial TLE as well as intra-operative intracranial electrode monitoring results of the medial temporal lobe structure discharge. The extent of resection of the temporal lobe was determined by intra-operative intracranial electrode monitoring of the extent of cortical involvement. If the Labbe vein was not exceeded, the discharge region was completely removed and if the discharge range was broad, the scope of temporal lobe resection did not exceed the Labbe vein. We generally used long-term intracranial electrode monitoring [18] to eliminate the cortical range involved in the discharge as much as possible. In the surgical procedure, we routinely used small incision [19] in the anterior temporal lobe and hippocampal amygdala resection to ensure the postoperative control of epilepsy. When the lesion was located in the lateral temporal lobe, or there was no clear evidence of hippocampal involvement, we used simple lesion or anterior temporal lobe resection. For patients who are diagnosed with TLE, early treatment is recommended $[20,21]$.

Pre-Operative Interictal Discharge Patterns
In our study, pre-operative interictal discharge was mainly observed as unilateral anterior (45.2\%) and bilateral (44.3\%) discharge, which together accounted for nearly $90 \%$ of cases. This indicates that the TLE discharge is likely to have uni- or bilateral involvement. In the unilateral anterior discharge, post-operative seizure-free rates were $96.1,92.2,88.2$, and $86.3 \%$ at $1,2,3$, and 4 years, respectively. All of these were higher than the general control rate. Post-operative seizure-free rates of the bilateral discharge were $69.6,67.4,60.9$, and $56.5 \%$ at $1,2,3$, and 4 years, respectively, representing a statistically significant difference. The pre- to post-operative EEG changes demonstrate that the EEG type progressed to normal and unilateral anterior discharge with the postoperative control of seizures. Thus, pre-operative interictal unilateral anterior discharge is associated with good prognosis, whereas bilateral discharge may have poor prognosis, as reported by others [11,22]. Our study found that for patients with normal MRI and relatively low seizure-free rates, the pre-operative discharge types were unilateral anterior (48.6\%) and bilateral (51.4\%) discharge. Thus, for patients with normal MRI results and 


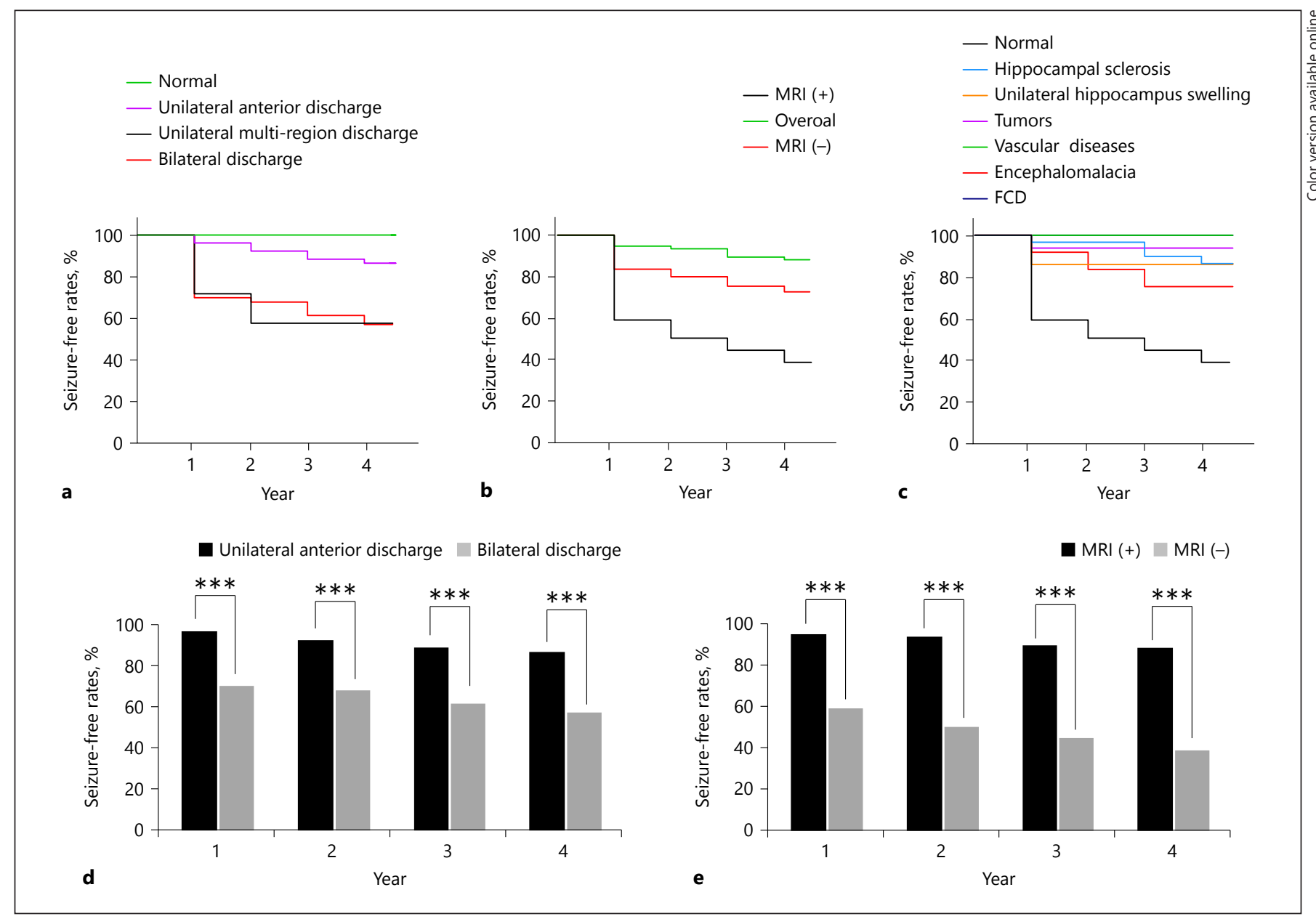

Fig. 5. a Trend of changes in the seizure-free rate in the post-operative follow-up, according to the pre-operative interictal EEG classification. $\mathbf{b}$ Change in the seizure-free rate in the post-operative follow-up of total cases and positive and negative magnetic resonance imaging (MRI) cases. c Trend of seizure-free rate in post-operative follow-up, according to the pre-operative MRI

confined unilateral anterior discharge pattern, surgical treatment could achieve better post-operative results [23]. We also suggest early surgical treatment for the normal MRI cases with unilateral anterior discharge pattern, because it may avoid nerve damage caused by long-term seizures [24] and the generalization of epileptic neural networks, including secondary contralateral discharges and independent seizures originating in the contralateral temporal lobe [25].

In the present study, when pre-operative MRI showed abnormal lesions, post-operative seizure-free rates at 1,2, 3 , and 4 years were $94.7,93.3,89.3$, and $88.0 \%$, respectively, which were higher than the overall control rate. In patients with abnormal MRI, unilateral anterior and bi- findings classification. $\mathbf{d}$ There was a statistically significant difference in the seizure-free rate between unilateral anterior and bilateral discharges at post-operative follow-up time points $(p<0.001)$. e There was a statistically significant difference in seizure-free rate in the post-operative follow-up between positive and negative MRI cases $(p<0.001)$.

lateral discharges accounted for larger proportions of pre-operation interictal discharge patterns in EEG. Although the 2 patterns differed in terms of prognosis, that of cases with abnormal MRI was generally better, suggesting that even if pre-operative interictal discharge pattern in the EEG varied, MRI abnormality could still predict the outcome of TLE patients [26]. We also found that although patients with abnormal MRI findings had better overall post-operative prognosis, post-operative seizures were sometimes observed along with hippocampal sclerosis, unilateral hippocampal swelling, tumor, and encephalomalacia. This could be related to seizures independently originating in the contralateral hippocampus, tumor recurrence, and insufficient resection of softening 
foci. Thus, pre-operative MRI imaging $[27,28]$ can be useful for identifying potential epileptic networks, and thereby improving the post-operative outcome of these patients.

\section{Conclusion}

In this study, we found that surgical treatment of TLE was highly effective. Regarding pre-operative interictal discharge patterns, patients with unilateral anterior discharge usually showed good prognosis; and those with MRI abnormalities showed good post-operative prognosis regardless of pre-operative interictal discharge patterns in EEG. Based on the follow-up data, we recommend that TLE patients with normal MRI and interictal discharge confined to the unilateral anterior region be treated by surgery to improve outcome.

\section{Acknowledgements}

Not applicable.

\section{Statement of Ethics}

Informed consent from each patient was obtained before the research. The use of the research protocol was approved by the Ethics and the Human Research Review Committee of Shengjing Hospital Affiliated to China Medical University.

\section{Disclosure Statement}

All authors consent to the publication of this manuscript. The authors declare that they have no competing interests.

\section{Funding Sources}

This research did not receive any specific grant from funding agencies in the public, commercial, or not-for-profit sectors.

\section{Author contributions}

All authors were involved in the study design, interpretation of results, and the reviewing and approval of the manuscript, and in the decision to submit the article for publication. All authors also confirm accountability for the accuracy and integrity of the work.

\section{References}

1 Ngugi AK, Bottomley C, Kleinschmidt I, Sander JW, Newton CR. Estimation of the burden of active and life-time epilepsy: a meta-analytic approach. Epilepsia. 2010 May; 51(5):883-90.

2 Fiest KM, Sauro KM, Wiebe S, Patten SB, Kwon CS, Dykeman J, et al. Prevalence and incidence of epilepsy: A systematic review and meta-analysis of international studies. Neurology. 2017 Jan;88(3):296-303.

3 Gu L, Liang B, Chen Q, Long J, Xie J, Wu G, et al. Prevalence of epilepsy in the People's Republic of China: a systematic review. Epilepsy Res. 2013 Jul;105(1-2):195-205.

4 Wiebe S. Definition of drug-resistant epilepsy: is it evidence based? Epilepsia. 2013 May; 54(suppl 2):9-12.

5 Larsson K, Eeg-Olofsson O. A population based study of epilepsy in children from a Swedish county. Eur J Paediatr Neurol. 2006 May;10(3):107-13.

6 Vermeulen L, van Loon J, Theys T, Goffin J, Porke K, Van Laere K, et al. Outcome after epilepsy surgery at the University Hospitals Leuven 1998-2012. Acta Neurol Belg. 2016 Sep;116(3):271-8.

7 Mohan M, Keller S, Nicolson A, Biswas S, Smith D, Osman Farah J, et al. The long-term outcomes of epilepsy surgery. PLoS One. 2018 May;13(5):e0196274.
8 Jayalakshmi S, Vooturi S, Vadapalli R, Somayajula S, Madigubba S, Panigrahi M. Outcome of surgery for temporal lobe epilepsy in adults - A cohort study. Int J Surg. 2016 Dec; 36(Pt B):443-7.

9 Kahane P, Bartolomei F. Temporal lobe epilepsy and hippocampal sclerosis: lessons from depth EEG recordings. Epilepsia. 2010 Feb; 51(suppl 1):59-62.

10 Sun Z, Zuo H, Yuan D, Sun Y, Zhang K, Cui $Z$, et al. Predictors of prognosis in patients with temporal lobe epilepsy after anterior temporal lobectomy. Exp Ther Med. 2015 Nov;10(5):1896-902.

11 Shin JH, Joo EY, Seo DW, Shon YM, Hong SB, Hong SC. Prognostic factors determining poor postsurgical outcomes of mesial temporal lobe epilepsy. PLoS One. 2018 Oct;13(10): e0206095.

12 Berkovic SF, McIntosh AM, Kalnins RM, Jackson GD, Fabinyi GC, Brazenor GA, et al. Preoperative MRI predicts outcome of temporal lobectomy: an actuarial analysis. Neurology. 1995 Jul;45(7):1358-63.

13 Wieser HG, Blume WT, Fish D, Goldensohn E, Hufnagel A, King D, et al.; Commission on Neurosurgery of the International League Against Epilepsy (ILAE). ILAE Commission Report. Proposal for a new classification of outcome with respect to epileptic seizures fol- lowing epilepsy surgery. Epilepsia. $2001 \mathrm{Feb}$ 42(2):282-6.

14 Deleo F, Garbelli R, Milesi G, Gozzo F, Bramerio M, Villani F, et al. Short- and long-term surgical outcomes of temporal lobe epilepsy associated with hippocampal sclerosis: relationships with neuropathology. Epilepsia. 2016 Feb;57(2):306-15.

15 Mathon B, Bielle F, Samson S, Plaisant O, Dupont $S$, Bertrand $A$, et al. Predictive factors of long-term outcomes of surgery for mesial temporal lobe epilepsy associated with hippocampal sclerosis. Epilepsia. 2017 Aug;58(8):1473-85.

16 Cendes F, Li LM, Watson C, Andermann F, Dubeau F, Arnold DL. Is ictal recording mandatory in temporal lobe epilepsy? Not when the interictal electroencephalogram and hippocampal atrophy coincide. Arch Neurol. 2000 Apr;57(4):497-500.

17 Wang MY, Wang J, Zhou J, Guan YG, Zhai F, Liu CQ, et al. Identification of the epileptogenic zone of temporal lobe epilepsy from stereo-electroencephalography signals: A phase transfer entropy and graph theory approach. Neuroimage Clin. 2017 Jul;16:184-95.

18 Tyrand R, Momjian S, Pollo C, Lysakowski C, Lascano AM, Vulliémoz S, et al. Continuous intraoperative monitoring of temporal lobe epilepsy surgery. Stereotact Funct Neurosurg. 2016;94(6):404-12. 
19 Alonso Vanegas MA, Lew SM, Morino M, Sarmento SA. Microsurgical techniques in temporal lobe epilepsy. Epilepsia. 2017 Apr; 58(suppl 1):10-8.

20 Engel J Jr, McDermott MP, Wiebe S, Langfitt JT, Stern JM, Dewar S, et al.; Early Randomized Surgical Epilepsy Trial (ERSET) Study Group. Early surgical therapy for drug-resistant temporal lobe epilepsy: a randomized trial. JAMA. 2012 Mar;307(9):92230.

21 Englot DJ, Chang EF. Rates and predictors of seizure freedom in resective epilepsy surgery: an update. Neurosurg Rev. 2014 Jul;37(3): 389-404.

22 Vollmar C, Stredl I, Heinig M, Noachtar S, Rémi J. Unilateral temporal interictal epileptiform discharges correctly predict the epilep- togenic zone in lesional temporal lobe epilepsy. Epilepsia. 2018 Aug;59(8):1577-82.

23 Burkholder DB, Sulc V, Hoffman EM, Cascino GD, Britton JW, So EL, et al. Interictal scalp electroencephalography and intraoperative electrocorticography in magnetic resonance imaging-negative temporal lobe epilepsy surgery. JAMA Neurol. 2014 Jun;71(6):702-9.

24 Shurtleff HA, Barry D, Firman T, Warner MH, Aguilar-Estrada RL, Saneto RP, et al. Impact of epilepsy surgery on development of preschool children: identification of a cohort likely to benefit from early intervention. J Neurosurg Pediatr. 2015 Oct;16(4):383-92.

25 Pustina D, Doucet G, Skidmore C, Sperling M, Tracy J. Contralateral interictal spikes are related to tapetum damage in left temporal lobe epilepsy. Epilepsia. 2014 Sep;55(9):1406-14.
26 Adry RA, Meguins LC, da Silva Júnior SC, Pereira CU, de Araújo Filho GM, Marques LH. Factors predicting the outcome following surgical treatment of mesial temporal epilepsy due to mesial temporal sclerosis. Acta Neurochir (Wien). 2016 Dec;158(12): 2355-63.

27 Sun Z, Yuan D, Sun Y, Zhang J, Zuo H, Zhang $\mathrm{K}$. Clinical curative effect analysis and predictors of prognosis in patients with temporal lobe epilepsy after anterior temporal lobectomy: results after five years. Chin Med J (Engl). 2014;127(14):2588-93.

28 Keller SS, Glenn GR, Weber B, Kreilkamp BA, Jensen JH, Helpern JA, et al. Preoperative automated fibre quantification predicts postoperative seizure outcome in temporal lobe epilepsy. Brain. 2017 Jan;140(1):68-82. 